\title{
On the composition of hybrid systems ${ }^{\star}$
}

\author{
Sébastien Bornot and Joseph Sifakis \\ Sebastien. Bornot@imag.fr Joseph.Sifakis@imag.fr \\ VerimaG, 2 rue Vignate, 38610 Gières, France
}

\section{Introduction}

Concurrent systems can be usually specified as systems of communicating processes obtained by composing sequential processes by means of binary parallel composition operators. The latter express process interaction in terms of action composition. Their semantics is usually defined by two types of rules.

- Synchronization rules that specify how an action of the product process is defined as the result of the (simultaneous) occurrence of two actions in two component processes.

- Interleaving rules, that specify how an action of a component process is an action of the product process. These rules allow some component processes to be idle while the others progress.

Combining synchronization and interleaving rules is essential for the specification of systems as process coordination requires both synchronization and waiting. However, their adequate combination must satisfy two conflicting requirements :

Deadlock-freedom : Deadlocks may appear in the product process as a result of enforcing synchronization, for instance, when two processes are at states from which only non matching synchronization actions can be performed. Such deadlocks can be avoided by using "escape" transitions generated by application of interleaving rules. However, the presence of both synchronization and interleaving actions may imply non maximal progress.

Maximal progress : When synchronization of two actions is possible, interleaving rules, used precisely to avoid deadlocks, may be applicable. Maximal progress means that synchronization is preferred to interleaving when both are possible. This is sometimes achieved by using restriction or hiding operators that prune out interleaving actions.

The above problems are amplified for timed or hybrid systems where time progress is synchronous and waiting times are bounded. This can be easily observed when hybrid specifications are obtained by adding timing constraints to untimed communicating systems specifications, as it has been pointed out in [SY96].

* in LNCS 1386, "Hybrid Systems : Computation and Control", Berkeley, April 1998, pp 49-63. 
In [SY96,BS97b] it is claimed that specifying time progress conditions independently from discrete transitions may be source of inconsistencies in specifications. We propose a model where time progress constraints are associated with actions and thus time progress is directly related with the ability of a system to perform actions. This model satisfies the property of time reactivity in the sense that if no action is enabled at a state, time can progress.

Following the process algebra approach, we consider discrete (untimed) systems represented as terms generated from a set of abstract actions by using operators such as prefixing, non deterministic choice and parallel composition. We extend the semantics of these operators to hybrid actions.

For a given abstract action $a$, a hybrid action extension of $a$, is defined as a triple $\left(g_{a}, d_{a}, f_{a}\right)$ where $g_{a}$ and $d_{a}$ are unary predicates and $f_{a}$ is a total function on a continuous set of states. The predicate $g_{a}$ is a guard characterizing the states from which $a$ is enabled while $d_{a}$ is a deadline satisfied by all the enabling states at which the action $a$ becomes urgent (time progress is stopped). The function $f_{a}$ represents the effect of the action when it is executed.

As usually, for a given n-ary operator op, the hybrid actions of the term $o p\left(t_{1}, \ldots, t_{n}\right)$ are obtained by composing the hybrid actions of the arguments $t_{i}$. We show that the semantics of operators on abstract actions can be extended to hybrid actions in different manners. The extensions have the same semantics for discrete transitions but may differ in urgency (ability to perform actions within a given delay).

We assume that parallel composition of two discrete systems can be expressed as the non-deterministic choice of terms starting with interleaving or synchronization actions (by means of some expansion theorem [BK85]). The expansion theorem is extended to hybrid actions in the following manner :

- To guarantee maximal progress, non-deterministic choice is replaced by priority choice that gives higher priority to synchronization actions over interleaving actions.

- Synchronization operators between abstract actions are extended to hybrid actions. The guard and the deadline resulting from the synchronization of two hybrid actions depend on the guards and deadlines of the synchronizing hybrid actions. We show that for hybrid actions different synchronization operations of practical interest can be defined by taking as synchronization guards and deadlines modal formulas. In particular, we identify three important synchronization modes : AND-synchronization where the guards of the synchronization action is the conjunction of the guards of the contributing actions. MAX-synchronization used to model synchronization with waiting and for which the synchronization action occurs as soon as all of the contributing actions have been completed. MIN-synchronization where the synchronization action occurs as soon as one of the contributing actions is completed. 
The paper is organized as follows. In section 2, we define hybrid extensions of discrete systems as a labeling homomorphism that extends prefixing and choice operators. Section 3 presents a framework for parallel composition of hybrid systems as an extension of parallel composition of untimed systems. For the three basic synchronization modes parallel composition rules are proposed that guarantee both local deadlock-freedom and maximal progress. We conclude by indicating possible application directions.

\section{Hybrid extensions of discrete systems}

We consider a simple (discrete) algebra of terms $S_{A}$ with prefixing and nondeterministic choice. We show that a hybrid extension of $S_{A}$ can be defined as a labeling of the underlying transition system associating with a state $s$, an evolution function $\triangleright_{s}$ and with any action $a$ a hybrid action $h(a)$.

\subsection{Discrete systems}

Consider the language of terms $S_{A}$ defined by the grammar

$$
s::=N i l|a . s| s+s
$$

where $N i l$ is a constant and $a \in A$, a set of atomic actions.

With a term of $S_{A}$ we associate transition relations subsets of $S_{A} \times A \times S_{A}$ defined by

a.s $\stackrel{a}{\rightarrow} s$

$s_{1} \stackrel{a}{\rightarrow} s_{1}{ }^{\prime}$ implies $s_{1}+s_{2} \stackrel{a}{\rightarrow} s_{1}{ }^{\prime}$ and $s_{2}+s_{1} \stackrel{a}{\rightarrow} s_{1}{ }^{\prime}$

We consider that + is an associative commutative operator with $\mathrm{Nil}$ as zero element. Any term $s$ is congruent (strongly bisimilar) to a term of the form :

$$
s=\sum_{i \in I} a_{i} . s_{i} \quad \text { (taken to be } N i l \text { if } I=\emptyset \text { ) }
$$

\subsection{Hybrid extension of $S_{A}$}

A hybrid extension of $S_{A}$ is defined as a pair $(V, h)$ where

$-V$ is a continuous state space isomorphic to $\mathbf{R}^{n}$ for some $n>0$

- $h$ is a labeling of $S_{A}$ such that :

- $h(s)=\left(s, \triangleright_{s}\right)$, where $\triangleright_{s}: V \times \mathbf{R}_{+} \rightarrow V$ is an evolution function. We write $v \triangleright_{s} t$ for $\triangleright_{s}(v, t)$. We require that $\triangleright_{s}$ is additive, i.e., $\forall v \in V \forall t_{1}, t_{2} \in \mathbf{R}_{+} \cdot v \triangleright_{s}\left(t_{1}+t_{2}\right)=\left(v \triangleright_{s} t_{1}\right) \triangleright_{s} t_{2}$.

- $h(a)=(a, g, d, f)$ where $g$ and $d$ are two unary predicates on $V$ and $f: V \rightarrow V$. We suppose that $d \Rightarrow g$. We call $g, d, f$ the guard, the deadline and the jump respectively of the hybrid action $h(a)$ associated with $a$. 
The hybrid extension of the term $s=\sum_{i} a_{i} . s_{i}$ is represented by the term $h(s)=\sum_{i} h\left(a_{i}\right) \cdot h\left(s_{i}\right)$.

We define hereafter the semantics of $h(s)$ in two steps. First, we associate transition relations with hybrid actions $h\left(a_{i}\right)$ on the continuous state space $V$. Then, we define the transition relation of the hybrid extension.

Definition 1. Let $b=(a, g, d, f)$ be a hybrid action associated with $a$ in some transition $s \stackrel{a}{\rightarrow} s^{\prime}$ of $S_{A}$. We define transition relations $\stackrel{t}{\rightarrow}$ for $t \in \mathbf{R}_{+}$and $\stackrel{a}{\rightarrow}$ for $a \in A$ subsets of $V \times V$ :

$-b: v \stackrel{t}{\rightarrow} v \triangleright_{s} t$ if $\forall t^{\prime}<t . \neg d\left(v \triangleright_{s} t^{\prime}\right)$

$-b: v \stackrel{a}{\rightarrow} f(v)$ if $g(v)$

The two relations describe the behavior of $b$ from a continuous state $v . b$ : $v \stackrel{t}{\rightarrow} v \triangleright_{s} t$ means that the execution of $b$ can be delayed for $t$ time units and $b: v \stackrel{a}{\rightarrow} f(v)$ represents the effect of a jump.

\section{Definition 2.}

The semantics of $h(s)=\sum_{i} b_{i} \cdot h\left(s_{i}\right)$ where $b_{i}=\left(a_{i}, g_{i}, d_{i}, f_{i}\right)$ and $h(s)=\left(s, \triangleright_{s}\right)$ is defined as a family of labeled transitions, subsets of $\left(S_{A} \times V\right) \times\left(A \cup \mathbf{R}_{+}\right) \times$ $\left(S_{A} \times V\right)$ by the rules

- If $b_{i}: v \stackrel{a_{i}}{\rightarrow} v_{i}$ then $(s, v) \stackrel{a_{j}}{\rightarrow}\left(s_{i}, v_{i}\right)$

- If $\forall i \in I$. $b_{i}: v \stackrel{t}{\rightarrow} v \triangleright_{s} t$ then $(s, v) \stackrel{t}{\rightarrow}\left(s, v \triangleright_{s} t\right)$.

Remark 3.

Notice that the projection of the transition relations on discrete state components agrees with the transition relations of the associated discrete system. This justifies the use of the term "extension".

Time can advance in $h(s)$ for $s=\sum_{i} a_{i} . s_{i}$ only if all the hybrid actions $h\left(a_{i}\right)$ agree to let time advance. This rule determines a time progress condition associated with $s$ similar to the "invariants" in $\left[\mathrm{ACH}^{+} 95\right]$ and "time progress conditions" in [KMP96]. Associating time progress with actions is an important feature of the presented model as it will be shown throughout the paper. For a given hybrid action, its guard characterizes the states from which the action is possible while its deadline characterizes the subset of the states where the action is enforced by stopping time progress.

The condition $d \Rightarrow g$ guarantees that if no action is enabled from a state then time can progress. In fact, time progress can stop only at states where a guard is enabled. Using terminology from synchronous language [JM94] we call this property time reactivity.

The relative position of $d$ with respect to the corresponding $g$ determines the urgency of an action. For a given $g$, the corresponding $d$ may take two extreme values: $d=g$ which means that the action is eager and $d=$ false which means that the action is lazy. A particularly interesting case is the one of delayable action where $d$ is the falling edge of $g$ (cannot be disabled without enforcing its execution) (figure 1). 


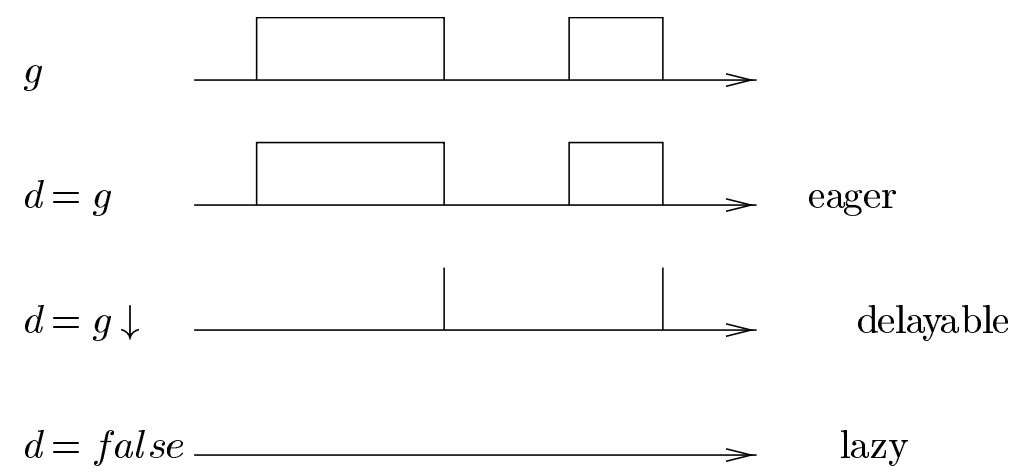

Fig. 1. using deadlines to specify urgency

\subsection{Choice operators}

Let $B=\left\{b_{i}\right\}_{i \in I}$ be a set of actions $b_{i}=\left(a_{i}, g_{i}, d_{i}, f_{i}\right)$ labeling transitions issued from a term with evolution function $\triangleright$. We use the modal operators $\diamond_{\leq k} p$ (eventually $p$ within $k$ ) and $\ominus_{\leq k} p$ (once $p$ since $k$ ) where $p$ is a unary predicate on $V$, and $k \in \mathbf{R}_{+} \cup\{\infty\}$.

$$
\begin{aligned}
& \diamond_{\leq k} p(v) \text { if } \exists t \in \mathbf{R}_{+} 0 \leq t \leq k . p(v \triangleright t) \\
& \diamond \leq k p(v) \text { if } \exists t \in \mathbf{R}_{+} 0 \leq t \leq k . \exists v^{\prime} \in V . v=v^{\prime} \triangleright t \wedge p\left(v^{\prime}\right)
\end{aligned}
$$

As usual, we write $\diamond p$ and $\diamond p$ for $\diamond_{\leq \infty} p$ and $\diamond \leq \infty p$ respectively, and $\square p$ and $\square p$ for $\neg \diamond \neg p$ and $\neg \diamond \neg p$ respectively.

We have already defined a non-deterministic choice operator $\sum_{i} b_{i} . s_{i}$ which combines the semantics of hybrid actions in a very simple manner. The discrete transition relation is the union of the discrete transition relations of the hybrid actions $b_{i}$ and the timed transition relation is the intersection of the timed transition relations of the $b_{i}$ 's. This semantics corresponds to a maximally urgent behavior in the sense that an action may occur when $\vee_{i} g_{i}$ holds and time progress stops as soon as $\vee_{i} d_{i}$ holds. In practice, it is often useful to define other choice operators with less prompt semantics ([BS97a]). We define a choice operator taking into account priorities between actions. Instead of considering non-deterministic choice between actions $b_{i}=\left(a_{i}, g_{i}, d_{i}, f_{i}\right)$, for $i=1,2$, one can consider that, for instance, $b_{2}$ has higher priority than $b_{1}$ which leads to restricting the guard and the deadline of $b_{1}$ to $g_{1}{ }^{\prime}$ and $d_{1}{ }^{\prime}$ respectively. One may take $g_{1}{ }^{\prime}=g_{1} \wedge \neg g_{2}$ and $d_{1}{ }^{\prime}=d_{1} \wedge g_{1}{ }^{\prime}$ to resolve conflicts between $b_{1}$ and $b_{2}$ in favor of $b_{2}$. This is a well-known manner to give priority to actions in untimed systems. However, for timed systems priority can concern not only instantaneous conflict resolution but also take into account possibility of waiting. For instance, if we take $g_{1}{ }^{\prime}=g_{1} \wedge \square \neg g_{2}$ and $d_{1}{ }^{\prime}=d_{1} \wedge g_{1}{ }^{\prime}$, we restrict the enabling states of $b_{1}$ to only those states from which $b_{2}$ will never be enabled. 
Definition 4. priority order

Consider the relation $<\subseteq A \times(\mathbf{N} \cup\{\infty\}) \times A$. We write $a_{1}<_{k} a_{2}$ for $\left(a_{1}, k, a_{2}\right) \in<$ and suppose that

$<_{k}$ is a partial order relation for all $k \in \mathbf{N} \cup\{\infty\}$

$a_{1}<k a_{2} \Rightarrow \forall k^{\prime}<k . a_{1}<k^{\prime} a_{2}$

$a_{1}<_{k} a_{2} \wedge a_{2}<_{l} a_{3} \Rightarrow a_{1}<_{k+l} a_{3}$

Property : The relation $a_{1} \ll a_{2}=\exists k a_{1}<_{k} a_{2}$ is an order relation.

Definition 5. priority choice operator

Given $<$, a priority order and $\left\{b_{i} \cdot s_{i}\right\}_{i \in I}$, a set of term, we define the priority choice operator $\sum_{<}$such that:

$$
\sum_{<}\left\{b_{i} . s_{i}\right\}_{i \in I}=\sum_{i \in I} b^{\prime}{ }_{i} . s_{i}
$$

where if $b_{i}=\left(a_{i}, g_{i}, d_{i}, f_{i}\right)$ then $b_{i}^{\prime}=\left(a_{i}, g^{\prime}{ }_{i}, d^{\prime}{ }_{i}, f_{i}\right)$ with $g_{i}^{\prime}=g_{i} \wedge \bigwedge_{a_{i}<{ }_{k} a_{j}} \neg \diamond_{\leq k} g_{j}$ and $d_{i}^{\prime}=d_{i} \wedge g_{i}^{\prime}$.

Notice that if $a_{i}<_{k} a_{j}$ then in $\sum b_{i}{ }_{i} \cdot s_{i}$ " $a_{j}$ has higher priority than $a_{i}$ in the interval $[0, k]$ " that is, $a_{i}$ is disabled if $a_{j}$ will be enabled within $k$ time units.

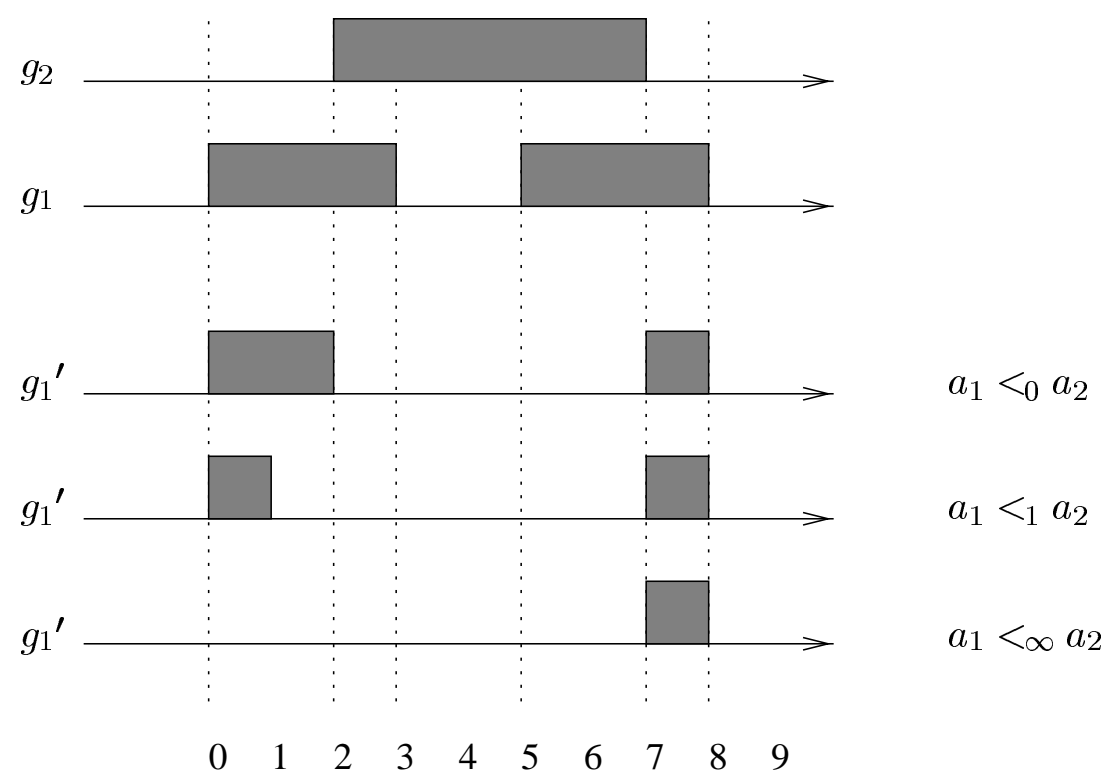

Fig. 2. Different priorities for $a_{2}$ over $a_{1}$ 
Consider the guards $g_{1}, g_{2}$ of the actions $a_{1}, a_{2}$. Figure 2 gives the guards $g_{1}^{\prime}$ obtained when $g_{1}$ is restricted by considering the priority orders $a_{1}<_{0} a_{2}$, $a_{1}<_{1} a_{2}, a_{1}<_{\infty} a_{2}$.

Proposition 6. The priority choice operators defined above satisfy the following properties.

$$
\begin{aligned}
& \text { 1. } \diamond g_{i} \Rightarrow \diamond\left(g^{\prime}{ }_{i} \vee \bigvee_{a_{i} \ll a_{j}} g_{j}\right) \\
& \text { 2. } \diamond \bigvee_{i \in I} g_{i}=\diamond \bigvee_{i \in I} g^{\prime}{ }_{i}
\end{aligned}
$$

The first property means that if action $a_{i}$ can occur in the non-prioritized choice then either $a_{i}$ can occur in the prioritized choice or some action of higher priority.

The second property is a consequence of the first and simply says that $\sum_{<}$ preserves (local) deadlock-freedom : if some action can be executed in the nonprioritized choice then some action can be executed in the prioritized choice and vice versa.

\section{Parallel composition}

In this section we define parallel composition operators by following the same approach as in the previous section. First, we show how parallel composition on hybrid systems can be defined as an extension of parallel composition on untimed systems. We thus obtain general composition rules for which some practically interesting cases are discussed later.

\subsection{Extending parallel composition from untimed to hybrid systems}

Untimed systems We consider a general framework for the composition of untimed terms. For this, we suppose that the vocabulary of actions $A$ contains a distinguished element $\perp$ and consider the set $A$ ı of the words generated from $A$ with a commutative operator $\mathrm{I}$ such that for all $a, a \mathrm{l} \perp=\perp$. The operator । is usually called communication function [BK85]. The words are used to represent synchronization actions that is, actions that result from the synchronous occurrence of atomic actions. $a_{1} \mid a_{2}=\perp$ means impossibility of synchronization.

In the sequel, we suppose that there are no other simplification rules for I but the rule for $\perp$ and that a word $a_{i} \mid a_{j}$ is given in reduced form.

Consider the language of terms $S_{A 1}$ defined by the grammar

$$
s::=s \in S_{A} \mid s \| s
$$


The semantics of the parallel composition operator is defined by the rules

$$
\begin{array}{r}
\left\{\begin{array}{l}
s_{1} \stackrel{a_{1}}{\rightarrow} s_{1}^{\prime} \\
s_{2} \stackrel{a_{2}}{\rightarrow} s_{2}^{\prime}
\end{array}\right\}, a_{1} \mid a_{2} \neq \perp \text { implies }\left\{\begin{array}{l}
s_{1}\left\|s_{2} \stackrel{a_{1} a_{2}}{\rightarrow} s_{1}{ }^{\prime}\right\| s_{2}{ }^{\prime} \\
s_{2}\left\|s_{1} \stackrel{a_{2} l a_{1}}{\rightarrow} s_{2}^{\prime}\right\| s_{1}{ }^{\prime}
\end{array}\right. \\
s_{1} \stackrel{a_{7}}{\rightarrow} s_{1}{ }^{\prime} \text { implies }\left\{\begin{array}{l}
s_{1}\left\|s_{2} \stackrel{a_{1}}{\rightarrow} s_{1}{ }^{\prime}\right\| s_{2} \\
s_{2}\left\|s_{1} \stackrel{a_{1}}{\rightarrow} s_{2}\right\| s_{1}{ }^{\prime}
\end{array}\right.
\end{array}
$$

$\|$ is a commutativeoperator that can be expressed in terms of non-deterministic choice. It is well-known that for $q_{1}=\sum_{i} a_{i} . s_{i}$ and $q_{2}=\sum_{j} a_{j} . s_{j}$,

$$
q_{1} \| q_{2}=\sum_{i} a_{i} \cdot\left(s_{i} \| q_{2}\right)+\sum_{j} a_{j} \cdot\left(s_{j} \| q_{1}\right)+\sum_{i, j} a_{i} a_{j} \cdot\left(s_{i} \| s_{j}\right)
$$

The first two summands start with interleaving actions while the last one starts with synchronization transitions (only terms such that $a_{i}{ }^{\prime} a_{j} \neq \perp$ appear).

Hybrid extension of $\boldsymbol{S}_{\boldsymbol{A}}$, For given $\left(V_{i}, h_{i}\right)$ hybrid extensions of $q_{i}$ for $i=1,2$, a hybrid extension $(V, h)$ for $q_{1} \| q_{2}$ is defined by :

$-V=V_{1} \times V_{2}$

- If $\tau_{i}=s_{i} \stackrel{a_{i}}{\rightarrow} s_{i}{ }^{\prime}$ is a transition of $q_{i}$ then $q_{1} \| q_{2}$ has transitions of the form $\tau=s_{1}\left\|s_{2} \stackrel{\lambda}{\rightarrow} s_{1}{ }^{\prime}\right\| s_{2}^{\prime}$ where $\lambda=a_{i}$ or $\lambda=\left.a_{1}\right|_{2}$. We take $h(\tau)=\left(s_{1} \|\right.$ $\left.s_{2}, \triangleright_{s_{1}} \times \triangleright_{s_{2}}\right) \stackrel{h(\lambda)}{\rightarrow}\left(s_{1}{ }^{\prime} \| s_{2}{ }^{\prime}, \triangleright_{s_{1}}{ }^{\prime} \times \triangleright_{s_{2}}{ }^{\prime}\right)$ where

- $h(\lambda)=h_{i}\left(a_{i}\right)$ if $\lambda=a_{i}$ and $h(\lambda)=h_{1}\left(a_{1}\right) ı h_{2}\left(a_{2}\right)$ if $\lambda=a_{1}$ । $a_{2}$ (we extend the communication function in an appropriate manner to hybrid actions, see below).

- $\triangleright_{s_{1}} \times \triangleright_{s_{2}}:\left(V_{1} \times V_{2}\right) \times \mathbf{R}_{+} \rightarrow V_{1} \times V_{2}$ is such that $\left(v_{1}, v_{2}\right)\left(\triangleright_{s_{1}} \times \triangleright_{s_{2}}\right) t=$ $\left(v_{1} \triangleright_{s_{1}} t, v_{2} \triangleright_{s_{2}} t\right)$.

This definition leads, by taking $b_{i}=h_{1}\left(a_{i}\right)$ and $b_{j}=h_{2}\left(a_{j}\right)$, to a scheme of expansion theorem for parallel composition where $\oplus$ and $\bigoplus$ are arbitrary choice operators (as defined in the previous section and in [BS97a]) :

$$
\begin{aligned}
h\left(q_{1} \| q_{2}\right)= & h_{1}\left(q_{1}\right)\left\|h_{2}\left(q_{2}\right)=\sum_{i} b_{i} \cdot h_{1}\left(s_{i}\right)\right\| \sum_{j} b_{j} \cdot h_{2}\left(s_{j}\right) \\
= & \bigoplus_{i} b_{i} \cdot\left(h_{1}\left(s_{i}\right) \| \sum_{j} b_{j} \cdot h_{2}\left(s_{j}\right)\right) \oplus \bigoplus_{j} b_{j} .\left(h_{2}\left(s_{j}\right) \| \sum_{i} b_{i} \cdot h_{1}\left(s_{i}\right)\right) \\
& \oplus \bigoplus_{i, j}\left(b_{i} \mid b_{j}\right) \cdot\left(h_{1}\left(s_{i}\right) \| h_{2}\left(s_{j}\right)\right)
\end{aligned}
$$

If $\oplus$ and $\oplus$ are non-deterministic choice operators then maximal progress is not guaranteed as an interleaving action may be executed when synchronization is possible. For this reason, we define parallel composition as the priority choice of the expanded terms with infinite priority to synchronization actions $b_{i} l b_{j}$ over the interleaving actions $b_{i}$ and $b_{j}$. This corresponds to priority choice for the 
minimal order $<$ such that $a_{i}<_{\infty} a_{i, j}$ and $a_{j}<_{\infty} a_{i, j}$ for any $i, j$. By using the notations

$$
\begin{aligned}
B= & \left\{b_{i} \cdot\left(h_{1}\left(s_{i}\right) \| \sum_{j} b_{j} \cdot h_{2}\left(s_{j}\right)\right)\right\}_{i} \cup\left\{b_{j} .\left(h_{2}\left(s_{j}\right) \| \sum_{i} b_{i} \cdot h_{1}\left(s_{i}\right)\right)\right\}_{j} \\
& \cup\left\{\left(b_{i} \mid b_{j}\right) .\left(h_{1}\left(s_{i}\right) \| h_{2}\left(s_{j}\right)\right)\right\}_{i, j}
\end{aligned}
$$

and $h_{1}\left(q_{1}\right)=\sum_{i} b_{i} . h_{1}\left(s_{i}\right)$ and $h_{2}\left(q_{2}\right)=\sum_{j} b_{j} . h_{2}\left(s_{j}\right)$, we have $h_{1}\left(s_{1}\right) \| h_{2}\left(s_{2}\right)=\sum_{<} B$ which is equivalent to

$$
\sum_{i} b_{i}{ }^{\prime} .\left(h_{1}\left(s_{i}\right) \| h_{2}\left(s_{2}\right)\right)+\sum_{j} b_{j}{ }^{\prime} \cdot\left(h_{2}\left(s_{j}\right) \| h_{1}\left(s_{1}\right)\right)+\sum_{i, j} b_{i} b_{j} .\left(h_{1}\left(s_{i}\right) \| h_{2}\left(s_{j}\right)\right)
$$
(figure 3)

In the above term, $b_{i}{ }^{\prime}, b_{j}{ }^{\prime}$ are the actions obtained by restricting $b_{i}$ and $b_{j}$ due

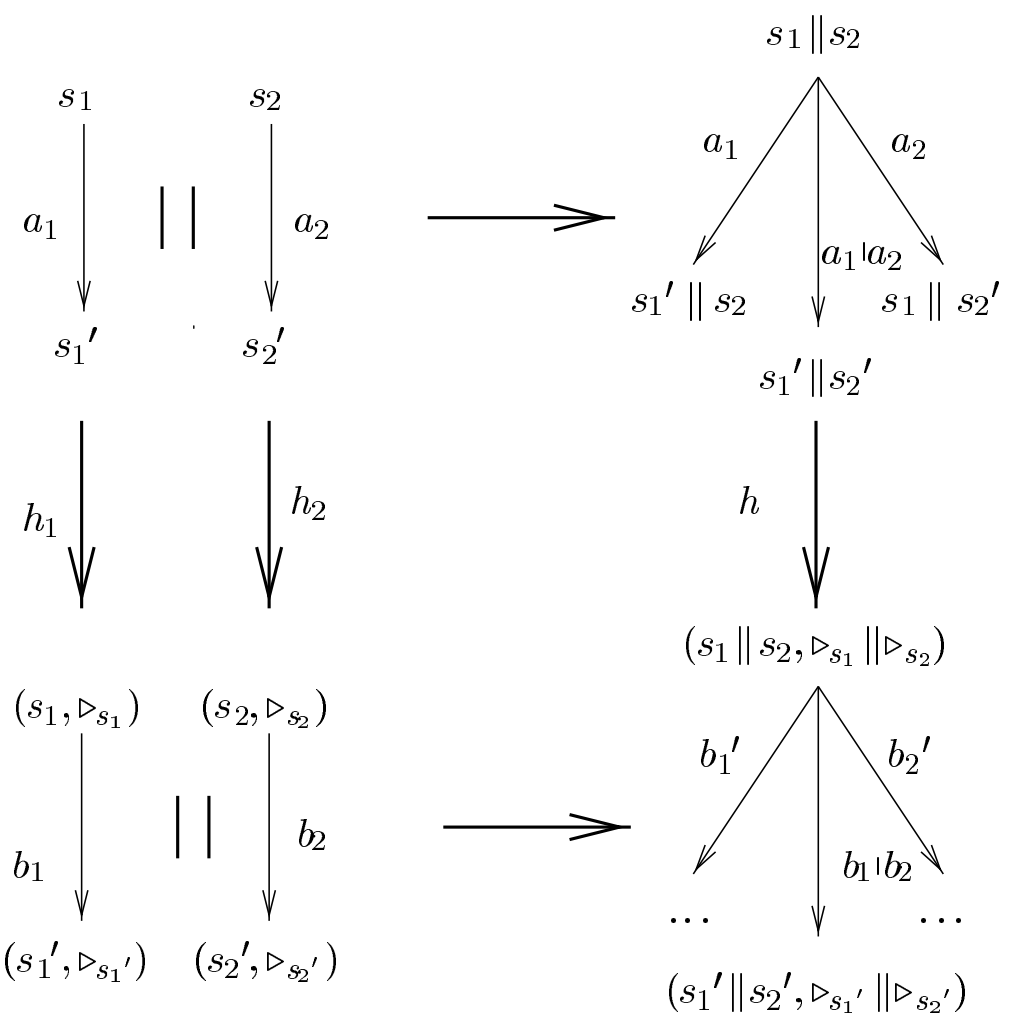

Fig. 3. Hybrid extension for parallel composition

to priority. We now define $b_{i} b b_{j}$.

Suppose that $h\left(a_{i}\right)=b_{i}=\left(a_{i}, g_{i}, d_{i}, f_{i}\right)$ for $i \in I$. If $a_{i} \mid a_{j}=\perp$ then we take $b_{i} \mid b_{j}=\perp$. Otherwise, we write $b_{i, j}=b_{i}\left|b_{j}=h\left(a_{i} \mid a_{j}\right)=h_{1}\left(a_{i}\right)\right| h_{2}\left(a_{j}\right)=$ $\left(a_{i} \backslash a_{j}, g_{i, j}, d_{i, j}, f_{i} \times f_{j}\right)$ where 
$f_{i} \times f_{j}: V_{1} \times V_{2} \rightarrow V_{1} \times V_{2}$ such that $\left(f_{i} \times f_{j}\right)\left(v_{1}, v_{2}\right)=\left(f_{i}\left(v_{1}\right), f_{j}\left(v_{2}\right)\right)$.

We propose in the next subsection a method for defining $g_{i, j}$ and $d_{i}, j$ by respecting the requirements $g_{i, j} \Rightarrow g_{i} \vee g_{j}$ and $d_{i, j} \Rightarrow d_{i} \vee d_{j}$ which mean that $b_{i, j}$ may be caused only by $b_{i}$ or $b_{j}$.

Proposition 7. If $g_{i, j} \Rightarrow g_{i} \vee g_{j}$, the above definition guarantees the following properties

1. local deadlock-freedom preservation that is,

$$
\diamond\left(\bigvee_{i \in I} g_{i} \vee \bigvee_{j \in J} g_{j}\right)=\diamond\left(\bigvee_{i \in I} g_{i}^{\prime} \vee \bigvee_{j \in J} g_{j}^{\prime} \vee \bigvee_{i \in I, j \in J} g_{i, j}\right)
$$

2. maximal progress that is, interleaving actions are executed only if synchronizations $b_{i, j}$ are disabled forever.

It is important to notice that these properties hold independently of the way the guards and deadlines of the synchronization actions are defined.

\subsection{Synchronization modes of hybrid actions}

Given two hybrid actions $b_{1}, b_{2}$ we define the guard $g_{1,2}$ and the deadline $d_{1,2}$ of the hybrid action $b_{1} b_{2}=\left(\left.a_{1}\right|_{a_{2}}, g_{1,2}, d_{1,2}, f_{1,2}\right)$ resulting from their apropriate synchronization.

Composition of guards : synchronization modes As already discussed in [SY96,BS97b], for timed and hybrid systems the guard $g_{1,2}$ can be in general a modal formula in terms of the guards $g_{1}$ and $g_{2}$. We consider in particular three important synchronization modes :

AND-synchronization requires that synchronization takes place only when both synchronized transitions can be executed. This means $g_{1,2}=g_{1} \wedge g_{2}$. Consider the example of two synchronizing actions with guards $g_{1}$ and $g_{2}$. Then, in general interleaving actions are needed to avoid deadlock. Their guards in this case will be $g_{1}{ }^{\prime}=g_{1} \wedge \square \neg\left(g_{1} \wedge g_{2}\right)$ and $g_{2}{ }^{\prime}=g_{2} \wedge \square \neg\left(g_{1} \wedge g_{2}\right)$.

MAX-synchronization requires that the first of the two synchronized actions that becomes enabled awaits for the other to become enabled. The enabling of the latest action triggers synchronization. A consequence of this assumption is that waiting may be unbounded. For a given execution trace, the time interval in which the synchronized action is enabled has as lower bound the maximum of the times they become enabled and as upper bound the maximum of the times they become disabled. The corresponding guard $g_{1,2}$ is defined by $g_{1,2}=\left(\diamond g_{1} \wedge g_{2}\right) \vee\left(g_{1} \wedge \diamond g_{2}\right)$. For this condition to express synchronization with waiting, it is necessary that if $s_{1}$ and $s_{2}$ are the source states of the transitions labeled by $b_{1}$ and $b_{2}$, these states should always be reached with values $v_{1}$ and $v_{2}$ such that $v_{i} \models_{s_{i}} \diamond g_{i}$ (remember that the meaning of $\diamond$ depends of the evolution 


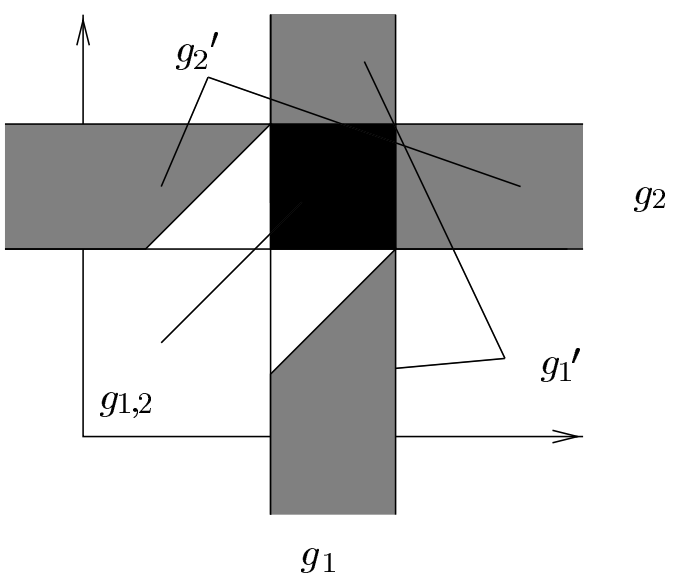

Fig.4. AND-synchronization

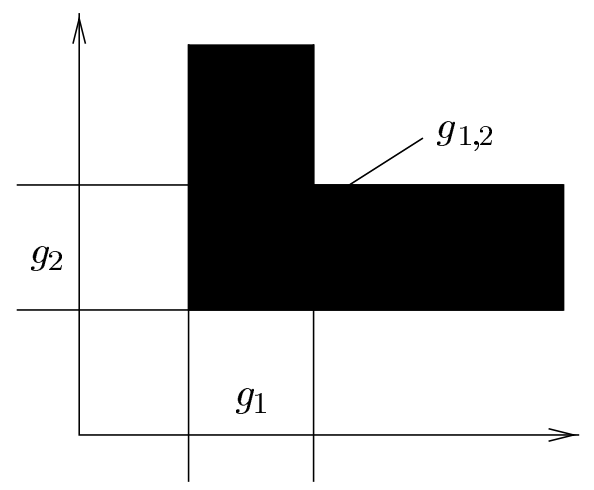

Fig. 5. MAX-synchronization 
function $\triangleright_{s_{i}}$ ). In the case where there are only two synchronizing actions whose guards are $g_{1}$ and $g_{2}$, the interleaving actions will have guards $g_{1}{ }^{\prime}=g_{1} \wedge \square \neg g_{1,2}$ and $g_{2}{ }^{\prime}=g_{2} \wedge \square \neg g_{1,2}$, which can be simplified into $g_{1}{ }^{\prime}=g_{1} \wedge \square \boxminus \neg g_{2}$ and $g_{2}{ }^{\prime}=g_{2} \wedge \square \square \neg g_{1}$.

MIN-synchronization is the dual of the previous synchronization mode, and it implies that the synchronization action $a_{1} \mid a_{2}$ can occur when one of the two synchronizing actions is enabled and the other will be eventually enabled. That is, synchronization may occur in a time interval whose lower bound is the minimum of the times they become enabled and the upper bound is the minimum of the times they become disabled. The corresponding guard $g_{1,2}$ is described by the formula $g_{1,2}=\left(\diamond g_{1} \wedge g_{2}\right) \vee\left(g_{1} \wedge \diamond g_{2}\right)$. In the case where

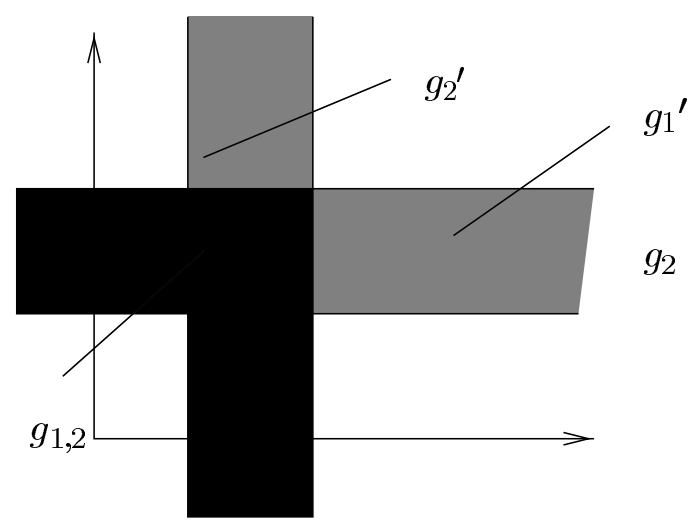

$g_{1}$

Fig. 6. MIN-synchronization

there are only two synchronizing actions with guards $g_{1}$ and $g_{2}$, the interleaving actions will have guards $g_{1}{ }^{\prime}=g_{1} \wedge \square \neg g_{1,2}=g_{1} \wedge \square \neg g_{2}$ and $g_{2}{ }^{\prime}=g_{2} \wedge \square \neg g_{1}$.

Composition of deadlines : typed transitions For two given hybrid actions $b_{1}=\left(a_{i}, g_{i}, d_{i}, f_{i}\right), i=1,2$ the deadline $d_{1,2}$ corresponding to $b_{1} b_{2}$ must satisfy the following condition

$$
d_{1,2} \Rightarrow g_{1,2} \wedge\left(d_{1} \vee d_{2}\right)
$$

Of course, the most urgent solution is to take $d_{1,2}=g_{1,2} \wedge\left(d_{1} \vee d_{2}\right)$ but this often leads to situations where the computed deadline $d_{1,2}$ does not correspond to the intuition [BS97a]. For this reason but also to introduce a simple model where deadlines are defined from guards by means of simple assumptions about urgency of the actions, we slightly modify our model. 
We suppose that the deadline $d_{i}$ of a hybrid action $b_{i}=\left(a_{i}, g_{i}, d_{i}, f_{i}\right)$ is defined by a function $\delta_{i}: 2^{V} \rightarrow 2^{V}$ such that $\delta_{i}\left(g_{i}\right)=d_{i}$.

An example of such a function is $\downarrow$ (falling edge). When $d_{i}=g_{i} \downarrow$ we have a delayable action according to our terminology. Another example is the identity function $\mathbf{1}=\lambda g . g$ which can be used to define eager actions. Finally, a trivial case is the function $\mathbf{0}=\lambda g$. fal se that allows to define lazy actions.

We call the function $\delta_{i} \in\{\mathbf{0}, \downarrow, \mathbf{1}\}$ types of the action. Types characterize the urgency of an action which is minimal for $\mathbf{0}$ and maximal for $\mathbf{1}$. Clearly, for synchronization between $b_{1}$ and $b_{2}$ it is necessary to define $\delta_{1,2}$ such that

$$
\underline{\delta_{1,2}\left(g_{1,2}\right) \Rightarrow g_{1,2} \wedge\left(\delta_{1}\left(g_{1}\right) \vee \delta_{2}\left(g_{2}\right)\right)}
$$

Proposition 8. The following table gives the most urgent type $\delta_{1,2}$ satisfying $(\alpha)$ for any mode (AND, MAX, MIN) in terms of $\delta_{1}, \delta_{2}$.

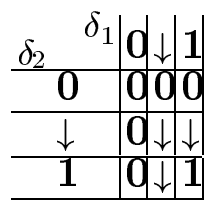

This result allows to reason only in terms of types of actions and drastically simplifies the general framework.

To complete the results we show that the type of a transition is preserved by priorities and thus the type of interleaving actions is the same as the type of the corresponding synchronizing transitions.

Proposition 9. If $d_{i}=g_{i}$ or $d_{i}=g_{i} \downarrow$ and $g_{i}{ }^{\prime}=g_{i} \wedge \square \neg g$ for some $g$, then $d_{i}{ }^{\prime}=d_{i} \wedge g_{i}{ }^{\prime}$ is such that $d_{i}{ }^{\prime}=g_{i}{ }^{\prime}$ or $d_{i}{ }^{\prime}=g_{i}{ }^{\prime} \downarrow$ respectively.

\section{Applications}

As an application of the above results, we define a parallel composition operator for typed hybrid actions that is, actions $b_{i}=\left(a_{i}, g_{i}, \delta_{i}, f_{i}\right)$ such that $\delta_{i} \in\{\mathbf{0}, \downarrow, \mathbf{1}\}$.

We suppose that for each pair of actions $\left(a_{1}, a_{2}\right)$ the synchronization mode is given. The resulting interleaving and synchronization actions depend on the synchronization mode. The synchronization action $b_{1,2}$ is $b_{1,2}=\left(a_{1} \mid a_{2}, g_{1,2}, \delta_{1,2}, f_{1,2}\right)$ where $g_{1,2}$ is defined in 3.2 according to the synchronization mode and $\delta_{1,2}$ is as specified in the table given in 3.2. The interleaving actions $b_{i}{ }_{i}$ are of the form $b_{i}^{\prime}=\left(a_{i}, g_{i}^{\prime}, \delta_{i}^{\prime}, f_{i}\right)$ where $g_{i}^{\prime}=g_{i} \wedge \square \neg g_{1,2}$ and $\delta_{i}^{\prime}=\delta_{i}$ (by proposition 9 ) for $i=1,2$.

Someapplications of this general framework can be found in [SY96] where it is shown that for timed Petri nets the underlying synchronization mode is MAXsynchronization. This allows to represent state machine decomposable timed Petri nets as the MAX-parallel composition of timed automata with delayable 
actions and makes possible the application of efficient timing analysis techniques to timed Petri nets.

An application domain for our results is modeling of multimedia systems where combinations of the different synchronization modes are necessary for a natural description of timing constraints. Several formalisms used in this area offer such possibilities. One of the most general seems to be the model of Time Stream Petri Nets, by Diaz et al[SDLdSS96]. These are Petri nets with interval time constraints where nine different synchronization modes can be associated with delayable transitions. It can be shown that the guards corresponding to the different synchronization modes can be expressed compositionally as modal formulas in terms of the guards of the components.

We are currently studying the application of the results to define the semantics of the language used in the MADEUS tool for the specification of multimedia documents [JLSIR97]. This language allows the description of timing constraints by means of logical and relational operators used to express causality and synchronization relations. The interesting fact is that very often a combination of the three synchronization types is necessary to specify coordination. The results of the study will be published in [BST97].

\section{Discussion}

We present a general framework for the composition of hybrid automata. We show that from elementary hybrid actions, choice and parallel composition, complex systems can be defined.

The main difference with other approaches is that we associate with actions time prog ress conditions which specify for how long an enabled action may wait. Time progress conditions at a given state depend on the urgency of the enabled actions.

The big variety of choice and parallel composition operators results from the different ways enabledness and urgency of components can be combined. Contrary to untimed systems, it is necessary to use modalities to express different kinds of composition that are of practical interest. However, for many tractable subclasses of hybrid automata modal operators can be eliminated, e.g. for linear hybrid automata $\left(\left[\mathrm{ACH}^{+} 95\right]\right)$. In that case, modalities are used just for notation convenience and do not modify the basic model.

Different choice operatorscan be expressed in terms of a basic non-deterministic choice operator which combines the behaviors of the contributing actions so as to obtain maximum urgency. Restricting guards to respect priorities leads to the definition of less prompt choice operators. Other kinds of restrictions remain to be investigated.

Priority choice plays an important role for the definition of a parallel composition operator that respects maximal progress and avoids deadlock by means of appropriate interleaving actions.

The proposed framework is very general. Validation by practice is necessary. It is important to notice that so far AND-synchronization has been used for timed 
process algebras and the different timed extensions of the language Lotos [LL95] as well as for timed and hybrid automata. MAX-synchronization is implicitly used in the different extensions of timed Petri nets.

We believe that AND-synchronization is more appropriate for responsive synchronization, where process coordination is supposed to be strong enough to impose that all the timing constraints of the contributing actions are respected. This is often the case for input/output, sender/receiver synchronization where one of the actions is not submitted to deadline constraints. For example, in the train-gate example often mentioned in the literature $\left[\mathrm{ACH}^{+} 95\right]$ communication between the two processes (train and gate) is responsive as the gate reacts to input signals sent by the train. Applying AND-synchronization to obtain the product automaton means that the deadlines and upper bounds of each process must be respected. On the contrary, synchronization between the gate process and a car stopped before the gate should allow for waiting and MAXsynchronization seems more appropriate in this case. We believe that MAXsynchronization should be used to extend parallel composition of asynchronous processes à la CSP. When a hybrid system is obtained as the hybrid extension of an untimed system of communicating automata, it is seems natural to use MAX-synchronization for actions that can wait indefinitely before synchronizing.

Finally, MIN-synchronization corresponds to a kind of (symmetric) interrupt and one can hardly imagine examples where the use of this synchronization mode alone suffices.

Acknowledgement : We thank S. Graf, S. Tripakis, E. Olive as well as M. Jourdan of the Opera project of INRIA for fruitful discussions about possible applications.

\section{References}

$\left[\mathrm{ACH}^{+} 95\right] \quad$ R. Alur, C. Courcoubetis, N. Halbwachs, T. Henzinger, P. Ho, X. Nicollin, A. Olivero, J. Sifakis, and S. Yovine. The algorithmic analysis of hybrid systems. Theoretical Computer Science, 138:3-34, 1995.

[BK85] J. A. Bergstra and J. W. Klop. Algebra of communicating processes with abstraction. Theoretical Computer Science, 37(1):77-121, May 1985. Fundamental studies.

[BS97a] S. Bornot and J. Sifakis. On the composition of hybrid systems (complete version). 1997.

[BS97b] S. Bornot and J. Sifakis. Relating time progress and deadlines in hybrid systems. In International Workshop, HART'97, pages 286-300, Grenoble, France, March 1997. Lecture Notes in Computer Science 1201, SpingerVerlag.

[BST97] S. Bornot, J. Sifakis, and S. Tripakis. Modeling Urgency in Timed Systems. To appear in COMPOS'97, LNCS, September 1997.

[JLSIR97] M. Jourdan, N. Layaida, L. Sabry-Ismail, and C. Roisin. An integrated authoring and presentation environment for interactive multimedia docu- 
ments. In 4th Conference on Multimedia Modeling, Singapore, November 1997. World Scientific Publishing.

[JM94] M. Jourdan and F. Maraninchi. Studying synchronous communication mechanism by abstractions. In IFIP Working Conference on Programming Concepts, Methods and Calculi, San Miniato, Italy, June 1994. Elsevier Science Publishers.

[KMP96] Y. Kesten, Z. Manna, and A. Pnueli. Verifying clocked transition systems. In School on Embedded Systems, Veldhoven, The Nederlands, November 1996.

[LL95] G. Leduc L. Léonard. An extended lotos for the design of real-time sys tems. In workshop DARTS'95, Bruxelles, Belgium, November 1995.

[SDLdSS96] P. Sènac, M. Diaz, A. Léger, and P. de Saqui-Sannes. Modeling logical and temporal synchronization in hypermedia systems. In Journal on Selected Areas in Communications, volume 14. IEEE, jan. 1996.

[SY96] J. Sifakis and S. Yovine. Compositional specification of timed systems. In 13th Annual Symposium on Theoretical Aspects of Computer Science, STACS'96, pages 347-359, Grenoble, France, February 1996. Lecture Notes in Computer Science 1046, Spinger-Verlag. 